\title{
O uso de plantas medicinais é efetivo para o controle de sintomas depressivos em adultos?: Uma revisão integrativa
}

\author{
Is the use of medicinal plants effective for the control of depressive symptoms in adults?: An \\ integrative review \\ ¿Es eficaz el uso de plantas medicinales para el control de los síntomas depresivos en adultos?: Una \\ revisión integradora
}

Recebido: 08/12/2021 | Revisado: 17/12/2021 | Aceito: 05/01/2022 | Publicado: 08/01/2022

\author{
Laiane Silva dos Santos \\ ORCID: https://orcid.org/0000-0002-6777-2246 \\ Universidade Federal do Sul da Bahia, Brasil \\ E-mail: laianee@live.com \\ Lucivania Cordeiro Silva \\ ORCID: https://orcid.org/0000-0003-0865-0844 \\ Universidade Federal do Sul da Bahia, Brasil \\ E-mail: lucii.vania@hotmail.com \\ Martone Moreira Conceição \\ ORCID: https://orcid.org/0000-0001-8145-4781 \\ Universidade Federal do Sul da Bahia, Brasil \\ E-mail: tony_moreira2007@ hotmail.com \\ Rosana Souza do Nascimento \\ ORCID: https://orcid.org/0000-0001-9311-5779 \\ Universidade Federal do Sul da Bahia, Brasil \\ E-mail: souzanana2008@gmail.com \\ Gisele Lopes de Oliveira \\ ORCID: https://orcid.org/0000-0001-8036-299X \\ Universidade Federal do Sul da Bahia, Brasil \\ E-mail: gisele.lopes@ufsb.edu.br \\ Maria Luiza Caires Comper \\ ORCID:https://orcid.org/0000-0003-2152-5263 \\ Universidade Federal do Sul da Bahia, Brasil \\ E-mail: maria.luiza@ufsb.edu.br
}

\begin{abstract}
Resumo
Este estudo buscou contribuir para a identificação e síntese das evidências de ensaios controlados randomizados sobre os efeitos do uso das plantas medicinais no controle dos sintomas depressivos em adultos. Trata-se de uma revisão integrativa, com busca realizada em bases de dados, utilizando os seguintes termos: (medicinal plants OR herbal plants), (depressive disorder OR depression), and (adults OR adult population). Foram incluídos: ensaios controlados randomizados; publicados em artigos originais disponíveis na íntegra; em língua inglesa ou portuguesa, e realizados com adultos de 18 a 59 anos. Foram excluídos os artigos duplicados e que não atenderam aos critérios de inclusão. A busca resultou em um total de 354 estudos, sendo 9 estudos incluídos para análise. A Crocus sativus (açafrão) foi a planta medicinal mais utilizada, com administração por meio de cápsulas em um período entre 4 e 12 semanas. $O$ uso das plantas medicinais mostrou efeitos positivos para redução da intensidade dos sintomas de depressão, melhora do desempenho da memória e do humor, e melhora na qualidade do sono. No entanto, este resultado deve ser interpretado com moderação, em razão do número de estudos e da qualidade metodológica.
\end{abstract}

Palavras-chave: Plantas medicinais; Depressão; Adulto.

\section{Abstract}

This study aims to contribute to the identification and synthesis of evidence from randomized controlled trials on the effects of the use of medicinal plants for the control of depressive symptoms in adults. This is an integrative review, with a search carried out in databases, using the following terms: (medicinal plants OR herbal plants), (depressive disorder OR depression), and (adults OR adult population). Randomized controlled trials; published in original articles available in full; in English or Portuguese, and performed with adults aged 18 to 59 years were included. Duplicate articles that did not meet the inclusion criteria were excluded. The search resulted in a total of 354 studies, with 9 studies included for analysis. Crocus sativus (saffron) was the most used medicinal plant, administered through capsules in a period between 4 and 12 weeks. The use of medicinal plants has shown positive effects in reducing the intensity of 
depression symptoms, improving memory and mood performance, and improving sleep quality. However, this result should be interpreted with moderation, due to the number of studies and methodological quality.

Keywords: Medicinal plants; Depression; Adults.

\section{Resumen}

Este estudio tiene como objetivo contribuir a la identificación y síntesis de evidencia de ensayos controlados aleatorios sobre los efectos del uso de plantas medicinales en el control de los síntomas depresivos en adultos. Se trata de una revisión integradora, con búsqueda realizada en bases de datos, utilizando los siguientes términos: (plantas medicinales o plantas herbáceas), (trastorno depresivo o depresión) y (adultos o población adulta). Se incluyeron los siguientes: ensayos controlados aleatorios; publicado en artículos originales disponibles en su totalidad; en inglés o portugués, y realizado con adultos de 18 a 59 años. Se excluyeron los artículos duplicados que no cumplieron con los criterios de inclusión. La búsqueda dio como resultado un total de 354 estudios, con 9 estudios incluidos para el análisis. Crocus sativus (azafrán) fue la planta medicinal más utilizada, administrada a través de cápsulas en un período de entre 4 y 12 semanas. El uso de plantas medicinales ha mostrado efectos positivos en la reducción de la intensidad de los síntomas de la depresión, mejorando el rendimiento de la memoria y el estado de ánimo y mejorando la calidad del sueño. Sin embargo, este resultado debe interpretarse con moderación, debido al número de estudios y la calidad metodológica.

Palabras clave: Plantas medicinales; Depresión; Adulto.

\section{Introdução}

A pandemia gerada pelo COVID-19 ocasionou uma mudança extensa no modo de vida de todos. Relações sociais impactadas, mudanças relativas ao modo de trabalho, luto e outros tantos fatores estressores, contribuíram para que as pessoas desenvolvessem uma série de sintomas depressivos. Esse contexto favoreceu o aumento da prevalência de tais condições, contribuindo de modo efetivo para a carga de doenças em todo o mundo. Estima-se que a prevalência mundial do transtorno depressivo no ano de 2020 tenha sido de 25\%, cerca de 7 vezes maior que aquela estimada no ano de 2017 (Bueno-Notivol et al, 2021).

Os transtornos depressivos incluem uma variedade de sintomas, tais como: tristeza, perda de interesse ou prazer, sentimentos de culpa ou baixa autoestima, sono ou apetite perturbados, sensação de cansaço e falta de concentração (WHO, 2017). A repercussão dessas manifestações impacta substancialmente a realização de atividades diárias, a capacidade para o trabalho, a participação social, o prazer envolvido em atividades de lazer, a utilização dos serviços de saúde e a saúde física (WHO, 2017; Kessler, Foster, Saunders, \& Stang, 1995; Johnson, Weissman, \& Klerman, 1992).

A alta prevalência da depressão tem atraído cada vez mais a atenção para a necessidade de cuidados em saúde. Diversos tratamentos têm sido descritos, como farmacológicos (antidepressivos inibidores seletivos de recaptação da serotonina, tricíclicos, lítio, mirtazapina, nefazodona, reboxetina, venlafaxina etc), psicoterapêuticos, compelementares e integrativos (Luo et al., 2018; Cleare et al. 2015; Fleck, Lafer, Sougey, Del Porto, Brasil \& Juruena et al., 2003). Dentro das práticas integrativas e complementares a utilização de plantas medicinais se destaca por valorizar saberes tradicionais, contribuir para promoção e autocuidado em saúde, apresentar menos efeitos colaterais, economia de custos diretos e indiretos para a saúde pública e contribuir para os sistemas locais de saúde (WHO, 2019; Almeida, 2019; WHO, 2013).

De fato, o uso de plantas medicinais para tratamento da depressão e de outras doenças aumentou nas últimas décadas (Yeung et al., 2018). Também houve avanços científicos importantes que permitiram o desenvolvimento de fitoterápicos seguros e eficazes, além de uma crescente tendência de busca por terapias menos agressivas destinadas ao atendimento primário à saúde (Bruning, Mosegui \& Vianna, 2012). Revisões anteriores documentaram efeitos de fitoterápicos para o tratamento da depressão clínicas específicas (Zeng et al., 2017) ou combinado com outras intervenções não-farmacológicas.

Assim, apesar dos avanços, ainda há limitação de estudos que descrevam os efeitos das plantas medicinais no tratamento da depressão (Cleare et al., 2015; Balbino \& Dias, 2010). Deste modo, o presente estudo buscou contribuir para a identificação e síntese das evidências de ensaios controlados randomizados sobre os efeitos do uso das plantas medicinais no controle dos sintomas depressivos em adultos. 


\section{Metodologia}

\section{Desenho de Estudo}

Trata-se de uma revisão integrativa da literatura, que se propôs a construir um panorama consistente, sintetizado e crítico das evidências científicas buscando responder a pergunta norteadora: Quais os efeitos do uso de plantas medicinais no tratamento da depressão em adultos?

O estudo cumpriu as diretrizes propostas por Whittemore \& Knafl (2005), tendo sido realizado em cinco etapas: (1) Elaboração da pergunta norteadora, delineada a partir da identificação do problema e da finalidade da pesquisa; (2) Estratégia de busca abrangente para seleção dos estudos, com definição dos critérios de inclusão e exclusão; (3) Extração das informações dos estudos selecionados; (4) Análise crítica dos estudo incluídos, considerando o desenho e a qualidade metodológica; (5) Interpretação e síntese dos resultados, a partir dos achados e suas conclusões.

\section{Estratégia de Busca}

A busca foi realizada nas bases de dados de Scientific Electronic Library Online (Scielo), U.S.National Library of Medicine (Pubmed), Biblioteca Virtual em Saúde (Bireme) e Literatura Latino-Americana do Caribe em Ciências da Saúde (Lilacs). Os termos de busca utilizados foram definidos a partir do acrônimo PICO. A estratégia de busca utilizada foi:("plants, medicinal"[MeSH Terms] OR ("plants"[All Fields] AND "medicinal"[All Fields]) OR "medicinal plants"[All Fields] OR ("medicinal"[All Fields] AND "plants"[All Fields]) OR (("herbal medicine"[MeSH Terms] OR ("herbal"[All Fields] AND "medicine"[All Fields]) OR "herbal medicine"[All Fields] OR "herbalism"[All Fields] OR "herbal"[All Fields] OR "herbals"[All Fields]) AND ("plant s"[All Fields] OR "planted"[All Fields] OR "planting"[All Fields] OR "plantings"[All Fields] OR "plants"[MeSH Terms] OR "plants"[All Fields] OR "plant"[All Fields]))) AND ("depressive disorder"[MeSH Terms] OR ("depressive"[All Fields] AND "disorder"[All Fields]) OR "depressive disorder"[All Fields] OR ("depressed"[All Fields] OR "depression"[MeSH Terms] OR "depression"[All Fields] OR "depressions"[All Fields] OR "depression s"[All Fields] OR "depressive disorder"[MeSH Terms] OR ("depressive"[All Fields] AND "disorder"[All Fields]) OR "depressive disorder"[All Fields] OR "depressivity"[All Fields] OR "depressive"[All Fields] OR "depressively"[All Fields] OR "depressiveness"[All Fields] OR "depressives"[All Fields])) AND ("adult"[MeSH Terms] OR "adult"[All Fields] OR "adults"[All Fields] OR "adult s"[All Fields]).

Foram incluídos ensaios clínicos randomizados que descrevessem o uso plantas medicinais, comparadas a nenhuma intervenção, intervenção mínima, ou qualquer tipo de intervenção; conduzidos com adultos de 18 a 59 anos e sem outras condições clínicas pré-diagnosticadas. Além destes, foram incluídos somente artigos originais disponíveis na íntegra, sem restrição de idioma, publicados nos últimos 10 anos (janeiro 2010 até janeiro 2021). Foram excluídos os estudos que não responderam à pergunta de pesquisa, duplicados, de revisão de literatura, narrativas, relatórios, monografias, teses e/ou aqueles não disponíveis em texto completo.

A seleção dos estudos foi realizada por dois pesquisadores independentes, em três etapas sequenciais: leitura de título, resumo e texto completo. Todos os textos selecionados na última etapa foram obtidos na íntegra e examinados de acordo com os critérios de inclusão e exclusão. Em caso de desacordo, os revisores resolveram por consenso ou por consulta a um terceiro pesquisador para arbitragem.

\section{Seleção dos estudos}

A seleção dos estudos foi realizada por dois pesquisadores independentes, em três etapas sequenciais: leitura de título, resumo e texto completo. Todos os textos selecionados na última etapa foram obtidos na íntegra e examinados de acordo com os critérios de inclusão e exclusão. Em caso de desacordo, os revisores resolveram por consenso ou por consulta a um terceiro pesquisador para arbitragem. 
Os critérios de inclusão foram: ensaios clínicos randomizados que descrevessem o uso plantas medicinais, comparadas a nenhuma intervenção, intervenção mínima, ou qualquer tipo de intervenção; conduzidos com adultos de 18 a 59 anos e sem outras condições clínicas pré-diagnosticadas. Além destes, foram incluídos somente artigos originais disponíveis na íntegra, sem restrição de idioma, publicados nos últimos 10 anos. Foram excluídos os estudos que não responderam à pergunta de pesquisa, duplicados, de revisão de literatura, narrativas, relatórios, monografias, teses e/ou aqueles não disponíveis em texto completo.

\section{Avaliação da Qualidade Metodológica}

A avaliação de qualidade metodológica foi realizada por meio da escala PEDro, na versão traduzida e adaptada para o português-brasileiro (Shiwa, Costa, Moser, Aguiar, \& Oliveira, 2011). A escala considera 11 critérios que avaliam a elegibilidade dos participantes, distribuição aleatória dos participantes por grupos, alocação secreta, similaridade dos grupos no que diz respeito aos indicadores de prognóstico, cegamento, dentre outros. A avaliação é feita através de uma pontuação de 0 a 10 por meio da soma do número de critérios considerados satisfatórios. Cada item cumprido pelo estudo corresponde a 1 (um) ponto, com exceção do primeiro critério, pois não possui pontuação atribuída.

\section{Extração e Síntese dos Dados}

O processo de extração de dados foi feito por dois avaliadores independentes, de modo similar ao descrito para o processo de seleção dos estudos. Em seguida, as informações extraídas foram discutidas e organizadas por consenso. Uma tabela de Excel foi elaborada para minimizar o risco de erros na transcrição, garantir precisão na checagem das informações e servir como registro. Os dados extraídos foram: autor, ano, título, população, nome da planta (família) utilizada na intervenção, medicamento utilizado no controle, ação, características da intervenção, dose/duração do tratamento e efeitos/resultados.

O processo de síntese das informações foi realizado por dois tipos de análise: descritiva e de conteúdo. A análise descritiva objetivou sintetizar as informações de caracterização dos estudos em dados de frequência absoluta e relativa. A análise de conteúdo, por sua vez, objetivou explorar e analisar as informações relacionadas aos efeitos das plantas medicinais no tratamento de depressão dos adultos.

\section{Resultados}

A busca resultou em um total de 354 estudos. Destes, 9 estudos foram excluídos após a verificação de duplicidade, 293 foram excluídos após emprego dos filtros e 38 excluídos após leitura de título e abstract. Assim, após a verificação dos critérios de elegibilidade foram selecionados 9 artigos para a realização do estudo (Figura 1). 
Figura 1 - Fluxograma dos estudos incluídos na revisão.

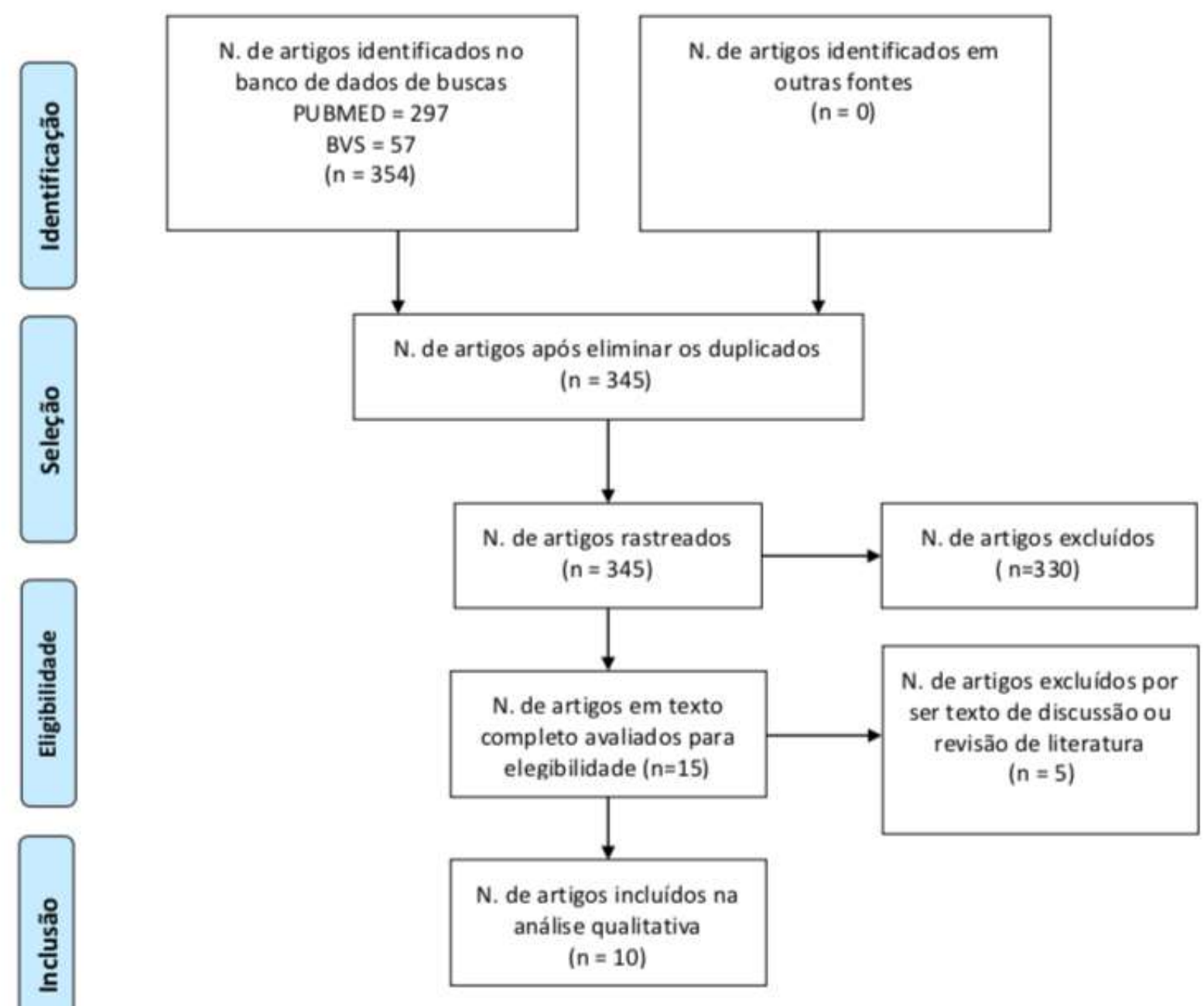

Fonte: Autores (2022).

A pontuação média total dos estudos pela escala PEDro foi de $6,56 \pm 1,33$. Somente 2 estudos ficaram com uma pontuação abaixo de 6, não ultrapassando 8 pontos (Tabela 1). O critério mais comprometido foi o da alocação dos sujeitos que não foi secreta, apenas um estudo fez essa alocação secretamente (Nematolahi, Mehrabani, Karami-Mohajeri \& Dabaghzadeh, 2018). Todos os estudos mediram os efeitos de tratamento com medidas de variabilidade. Apenas um estudo (Panahi, Badeli, Karami, Badeli \& Sahebkar, 2015) não cumpriu o critério de cegamento dos pacientes e terapeutas. 
Tabela 1 - Qualidade metodológica dos estudos incluídos na revisão.

Autor (ano)

Qualidade Metodológica - Escala PEDro

\begin{tabular}{|c|c|c|c|c|c|c|c|c|c|c|c|c|}
\hline & $\begin{array}{l}\text { Crité } \\
\text { rio } 1\end{array}$ & $\begin{array}{l}\text { Crité } \\
\text { rio } 2\end{array}$ & $\begin{array}{l}\text { Crité } \\
\text { rio } 3\end{array}$ & $\begin{array}{l}\text { Crité } \\
\text { rio } 4\end{array}$ & $\begin{array}{l}\text { Crité } \\
\text { rio } 5\end{array}$ & $\begin{array}{l}\text { Crité } \\
\text { rio } 6\end{array}$ & $\begin{array}{l}\text { Crité } \\
\text { rio } 7\end{array}$ & $\begin{array}{l}\text { Crité } \\
\text { rio } 8\end{array}$ & $\begin{array}{l}\text { Crité } \\
\text { rio } 9\end{array}$ & $\begin{array}{l}\text { Crité } \\
\text { rio } 10\end{array}$ & $\begin{array}{l}\text { Crité } \\
\text { rio } 11\end{array}$ & $\begin{array}{r}\text { Tot } \\
\text { al }\end{array}$ \\
\hline $\begin{array}{l}\text { Araj-Khodaei et al. } \\
(2020)\end{array}$ & 1 & 0 & 0 & 1 & 0 & 0 & 0 & 1 & 0 & 1 & 1 & 4 \\
\hline $\begin{array}{l}\text { Anushiravani et al. } \\
\text { (2019) }\end{array}$ & 1 & 1 & 0 & 1 & 1 & 0 & 0 & 0 & 1 & 1 & 0 & 5 \\
\hline Kyrou et al. (2017) & 1 & 1 & 0 & 1 & 1 & 0 & 0 & 0 & 1 & 1 & 1 & 7 \\
\hline $\begin{array}{l}\text { Nematolahi et al. } \\
(2017)\end{array}$ & 1 & 1 & 1 & 1 & 1 & 0 & 1 & 1 & 0 & 1 & 0 & 7 \\
\hline Kolouri et al. (2016) & 1 & 0 & 0 & 1 & 1 & 0 & 0 & 0 & 1 & 1 & 1 & 5 \\
\hline Mazidi et al. (2016) & 1 & 1 & 0 & 1 & 1 & 0 & 1 & 0 & 1 & 1 & 1 & 7 \\
\hline $\begin{array}{l}\text { Di Pierro; Risso; } \\
\text { Settembre (2016) }\end{array}$ & 1 & 0 & 0 & 1 & 0 & 0 & 0 & 1 & 1 & 0 & 1 & 4 \\
\hline Panahi et al. (2015) & 1 & 0 & 0 & 1 & 0 & 0 & 0 & 0 & 1 & 1 & 1 & 4 \\
\hline $\begin{array}{l}\text { Sahraian et al. } \\
(2015)\end{array}$ & 1 & 1 & 0 & 1 & 1 & 0 & 0 & 1 & 1 & 1 & 0 & 6 \\
\hline Talaei et al. (2015) & 1 & 1 & 0 & 1 & 1 & 0 & 0 & 1 & 1 & 1 & 1 & 7 \\
\hline
\end{tabular}

Legenda: Item 1= O objetivo do estudo foi claramente definido?; Item 2= Houve descrição dos métodos utilizados para o ensino remoto?Item 3 =Os métodos utilizados atingem o objetivo do estudo?; Item 4= Há descrição dos participantes/estudantes?Item 5=Há descrição de como os resultados foram coletados ?; Item $6=$ Os resultados foram coletados corretamente?; Item 7= As questões éticas foram respeitadas?; Item 8= Os resultados foram analisados corretamente?; Item $9=$ Os resultados foram apresentados corretamente?; Item $10=$ Os achados são relevantes ?. $1=$ Item atendido; $0=$ item não atendido ou não descrito. Fonte: Autores .

Em relação às plantas medicinais, foi possível levantar o uso de 8 espécies e de uma microalga no tratamento da depressão. A Crocus sativus (açafrão) foi a planta medicinal mais utilizada (Anushiravani, Manteghi, Taghipur \& Eslami, 2019; Mazidi et al., 2016; Sahraian, Jelodar, Javid, Mowla, \& Ahmadzadeh, 2016; Talaei, Moghadam, Tabassi \& Mohajeri 2015), seguida da Melissa Officinalis (erva-cidereira) (Araj-Khodaei et al., 2020; Anushiravani et al.,2019). A forma mais utilizada para administração foi por meio de cápsulas (Araj-Khodaei et al., 2020; Nematolahi et al., 2017; Kyrou, Christou, Panagiotakos, Stefanaki, Skenderi, Katsana \& Tsigos, 2017; Mazidi et al., 2016; Saharaian et al., 2016; Kolouri, Firoozabadi, Salehi, Zarshenas, Dastgheib, Heydari, \& Rezaeizadeh, 2016). A duração de uso das plantas medicinais variou entre 4 e 12 semanas (Tabela 2). 
Tabela 2 - Descrição das plantas medicinais, doses e efeitos obtidos por ensaios controlados randomizados em teste com adultos com sintomas depressivos.

\begin{tabular}{|c|c|c|c|c|c|c|}
\hline Autor & $\begin{array}{l}\text { Espécie (nome } \\
\text { popular) }\end{array}$ & $\begin{array}{l}\text { Medicamento } \\
\text { (controle) }\end{array}$ & $\begin{array}{l}\text { Características da } \\
\text { intervenção }\end{array}$ & $\begin{array}{l}\text { Duração do } \\
\text { tratamento }\end{array}$ & $\begin{array}{l}\text { Medida do } \\
\text { desfecho }\end{array}$ & Efeitos resultados \\
\hline \multicolumn{7}{|c|}{ Plantas medicinais X Medicamentos antidepressivos } \\
\hline $\begin{array}{l}\text { Araj- } \\
\text { Khodaei et } \\
\text { al. }(2020)\end{array}$ & $\begin{array}{l}\text { Melissa } \\
\text { officinalis L. } \\
\text { (erva-cidreira); } \\
\text { Lavandula } \\
\text { angustifolia } \\
\text { Mill. (lavanda) }\end{array}$ & Fluoxetina & $\begin{array}{l}\text { Os participantes } \\
\text { foram divididos em } \\
3 \text { grupos. A } \\
\text { fluoxetina foi o } \\
\text { grupo controle. } \\
\text { Cápsulas de cada } \\
\text { substância estudada } \\
\text { foram preparadas } \\
\text { com } 500 \text { mg de pó. } \\
\text { A dose foi de: } M \text {. } \\
\text { officinalis }(2 \mathrm{~g}) \text { ou } \\
\text { L. angustifolia }(2 \mathrm{~g}) \\
\text { ou fluoxetina }(10 \\
\text { mg). }\end{array}$ & 8 semanas & $\begin{array}{l}\text { Escala de } \\
\text { Avaliação de } \\
\text { Hamilton } \\
\text { para } \\
\text { Depressão } \\
\text { (HAM-D) }\end{array}$ & 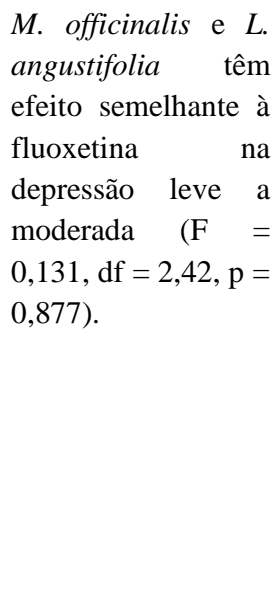 \\
\hline $\begin{array}{l}\text { Anushirav } \\
\text { ani et al. } \\
(2019)\end{array}$ & $\begin{array}{l}\text { Echium } \\
\text { amoenum Fisch. } \\
\text { \& C.A.Mey.; } \\
\text { Melissa } \\
\text { officinalis L. } \\
\text { (erva-cidreira); } \\
\text { Crocus sativus } \\
\text { L. (açafrão) }\end{array}$ & Citalopram & $\begin{array}{l}\text { Citalopram foi } \\
\text { prescrito para usar } \\
20 \mathrm{mg} \text { por dia no } \\
\text { grupo controle. O } \\
\text { grupo intervenção } \\
\text { recebeu E.amoenum } \\
\text { em forma de xarope } \\
\text { (30 mL duas vezes } \\
\text { por dia, uma hora } \\
\text { antes da refeição). } \\
\text { Cada } 30 \text { mL do } \\
\text { xarope continha } 3 \mathrm{~g} \\
\text { de flores de E. } \\
\text { amoenum, } 1,5 \mathrm{~g} \\
\text { folhas } \text { M. officinalis } \\
\text { e } 150 \text { mg de estigma } \\
\text { de C. sativus. }\end{array}$ & 8 semanas & $\begin{array}{l}\text { Escala de } \\
\text { Avaliação de } \\
\text { Hamilton } \\
\text { para } \\
\text { Depressão } \\
\text { (HAM-D) }\end{array}$ & $\begin{array}{lr}\text { Os pacientes } & \text { em } \\
\text { ambos os grupos de } \\
\text { citalopram } \text { e } \\
\text { amoenum } \\
\text { mostraram redução } \\
\text { notável } & \text { nas } \\
\text { pontuações } & \text { do } \\
\text { questionário } & \text { de } \\
\text { Hamilton. } 52 \% \text { dos } \\
\text { pacientes tiveram } \\
\text { efeitos colaterais } \\
\text { várias complicações } \\
\text { no grupo } \\
\text { citalopram, do } \\
\text { enquanto apenas } \\
\text { 12\% dos pacientes } \\
\text { no } \\
\text { intervenção } \\
\text { relataram } \\
\text { complicações. }\end{array}$ \\
\hline $\begin{array}{l}\text { Di Pierro } \\
\text { et } \quad \text { al. } \\
(2018)\end{array}$ & $\begin{array}{l}\text { Hypericum } \\
\text { perforatum L. } \\
\text { convencional } \\
\text { (C-Hp) (Erva- } \\
\text { de-são-joão) }\end{array}$ & $\begin{array}{l}\text { Multi-fracionado } \\
\text { de Hypericum } \\
\text { perforatum } \\
\text { (M-Hp) (IperiPlex } \\
\text { (B) }\end{array}$ & $\begin{array}{l}\text { Os pacientes } \\
\text { receberam } \\
\text { comprimidos/dia de } \\
\text { Nervaxon }{ }^{\circledR} \text { (Fidia, } \\
\text { Itália) ou IperiPlex } \\
\circledR \text { (PharmExtracta, } \\
\text { Itália). Os dois } \\
\text { produtos continham } \\
\text { a mesma quantidade } \\
\text { (300 mg / tab) de } \\
\text { extrato de H. } \\
\text { perforatum e eram } \\
\text { tomados duas vezes } \\
\text { por dia às } 8 \mathrm{~h} 00 \text { e } \\
\text { 20h00 com o } \\
\text { estômago vazio. }\end{array}$ & 12 meses & $\begin{array}{l}\text { Autoavaliaçã } \\
\text { o da } \\
\text { Depressão de } \\
\text { Zung }\end{array}$ & $\begin{array}{l}\text { O tratamento com } \\
\text { multi-fracionado de } \\
\text { H. perforatum foi } \\
\text { duas vezes mais } \\
\text { eficaz do que o } \\
\text { tratamento com } H \text {. } \\
\text { perforatum } \\
\text { convencional. Não } \\
\text { foram relatados } \\
\text { efeitos colaterais. }\end{array}$ \\
\hline
\end{tabular}




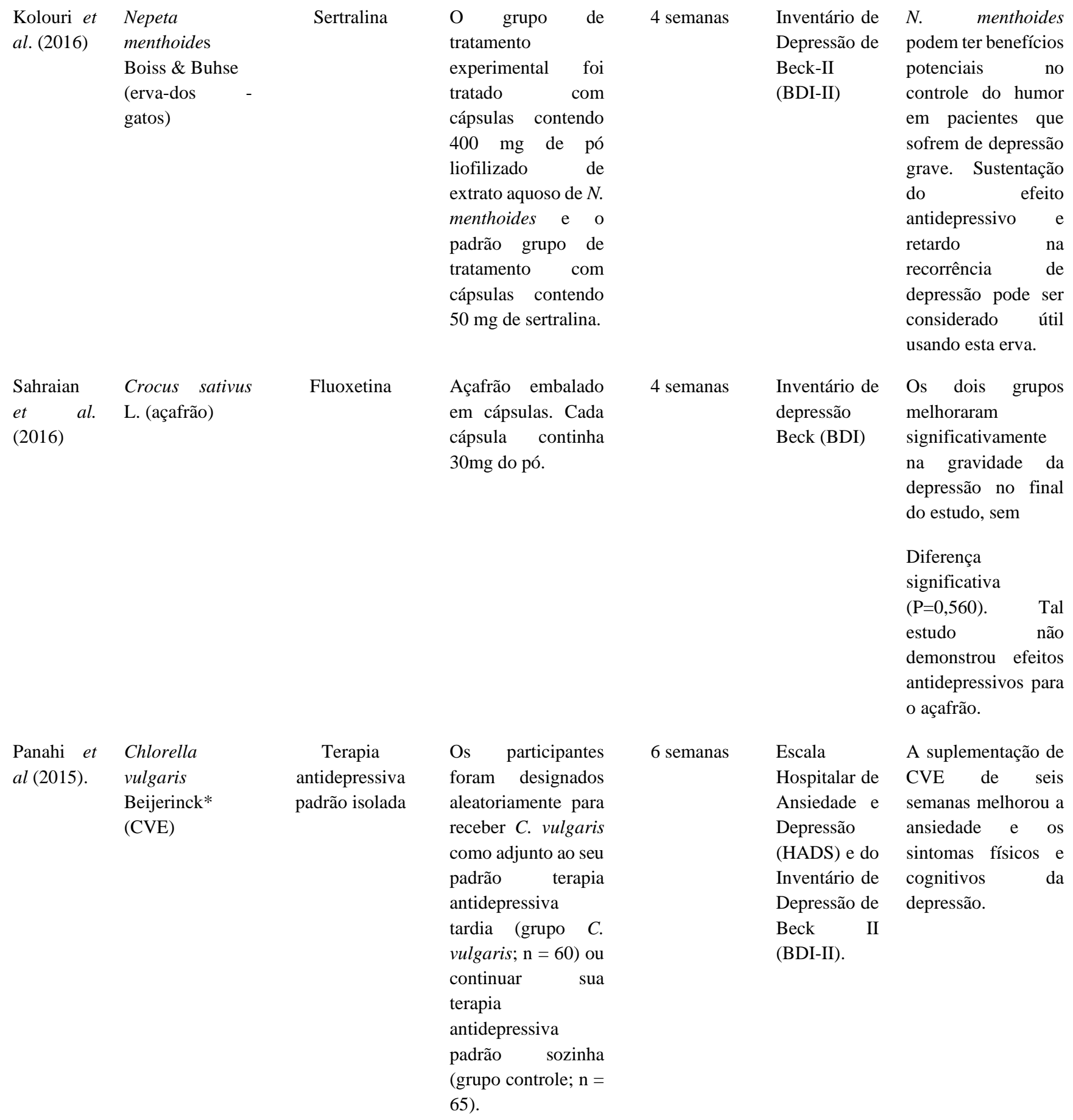

Plantas medicinais X placebo

\begin{tabular}{|c|c|c|c|c|c|c|}
\hline $\begin{array}{l}\text { Kyrou et } \\
\text { al. (2017) }\end{array}$ & $\begin{array}{l}\text { Humulus } \\
\text { lupulus } \\
\text { L.(lúpulo) }\end{array}$ & Placebo & $\begin{array}{l}\text { Foram } \\
\text { administrados } \\
\text { Lúpulo de } \\
\text { melcalina ou } \\
\text { placebo; duas } \\
\text { cápsulas de } 0,2 \mathrm{~g} \\
\text { uma vez } \\
\text { diariamente, } \\
\text { separados por um } \\
\text { intervalo de } 2\end{array}$ & 4 semanas & $\begin{array}{l}\text { Depression } \\
\text { Anxiety } \\
\text { Stress Scale- } \\
21\end{array}$ & $\begin{array}{l}\text { Os escores de } \\
\text { ansiedade, } \\
\text { depressão e estresse } \\
\text { DASS-21 } \\
\text { (Depression, } \\
\text { Anxiety and Stress } \\
\text { Scale) foram } \\
\text { significativamente } \\
\text { diminuídos. }\end{array}$ \\
\hline
\end{tabular}

semanas. 


$\begin{array}{llll}\text { Nematolah } & \text { Rosmarinus } & \text { Placebo (amido) } & \text { Partes aéreas secas } \\ \text { i et al. } & \begin{array}{l}\text { officinalis } \\ \text { (alecrim) }\end{array} & \text { em pó de alecrim } \\ (2017) & & \text { (no grupo de } \\ & & \text { alecrim) e amido } \\ & \text { como placebo (no } \\ & \text { grupo de controle) } \\ & \text { foram } \\ & \text { administradas como } \\ & \text { uma cápsula de } 500 \\ & \text { mg duas vezes ao } \\ & \text { dia. }\end{array}$
$\begin{array}{lll}\text { Mazidi et } & \text { Crocus sativus } & \text { Placebo } \\ \text { al. (2016) } & \text { L. (açafrão) } & \end{array}$

4 semanas

60 pacientes adultos 12 semanas
receberam 50 mg de
açafrão e de placebo
em cápsula, duas
vezes ao dia durante
$12 \quad$ semanas.54
completaram o
estudo.

A versão
persa
validada de
Prospectiva e
Retrospectiv
a
Questionário
de memória
(PRMQ),
Escala
Hospitalar de
Ansiedade e
Depressão
(HADS), A
versão persa
validada da
Qualidade do
Sono de
Pittsburgh
(PSQI).

Inventário de depressão Beck (BDI), Questionário do Inventário de Ansiedade (BAI).
Efeitos significativos na melhoria do desempenho da memória, reduzindo ansiedade e depressão, e melhorando a qualidade do sono. $\mathrm{O}$ efeito do tempo foi um fator estatisticamente significativo para todas as escalas e subescalas.
Os suplementos de açafrão tiveram um efeito significativo sobres as pontuações com base no questionário de Beck Depression Inventory (BDI) e Beck Anxiety Inventory (BAI) questionnaire, dos sujeitos em comparação com placebo no ponto de tempo de 12 semanas $(\mathrm{p}<0,001)$.

\footnotetext{
* Chlorella vulgaris - micro-alga. Fonte: Autores.
}

Os desfechos relacionados aos sintomas depressivos foram avaliados por questionários auto respondidos. O Beck Depression Inventory (BDI) foi descrito em cinco estudos (Kolouri et al., 2016; Saharaian et al., 2016; Mazidi et al., 2016; Panahi et al., 2015; Talaei et al., 2015). A Escala de Avaliação de Depressão de Hamilton (HAM-D) foi utilizada em dois estudos (Araj-khodaei et al., 2020; Anushiravani et al.,2019). Além destes, também foram empregadas a Escala de Ansiedade Depressão e Estresse-21(EADS-21)(Kyrou et al., 2017), a versão persa do Questionário de Memória Prospectiva e Retrospectiva (PRMQ), a Escala Hospitalar de Ansiedade e Depressão (HADS) e versão persa da Qualidade do Sono de Pittsburgh (PSQI) (Nematollahi et al., 2017), o Questionário do Inventário de Ansiedade (BAI) (Talaei et al., 2015; Mazidi et al., 2016), o Questionário geral de saúde (GHQ) e o Questionário de transtorno de humor (MDQ) (Talaei et al., 2015).

Os efeitos das plantas medicinais foram medidos comparando-as com outros medicamentos antidepressivos, com placebo e como adição ao medicamento antidepressivo. Assim, 3 estudos compararam as plantas medicinais a medicamentos antidepressivos tradicionais, como a fluoxetina, citalopram e sertralina (Araj-Khodaei et al., 2020; Anushiravani et al.,2019; Kolouri et al., 2016). Estes estudos mostraram que as plantas medicinais foram eficazes em reduzir/controlar os sintomas depressivos (Araj-Khodaei et al., 2020; Anushiravani et al.,2019; Kolouri et al., 2016). A M. officinalis (erva-cidreira) e 
Lavandula angustifolia (lavanda) demonstraram terem um efeito semelhante à fluoxetina na depressão leve a moderada pela HAM-D (Araj-Khodaei et al. 2020). O uso de Nepeta menthoides (erva-dos -gatos) comparada a sertralina mostrou potenciais benefícios no controle de humor em pacientes com depressão grave além da sustentação do efeito antidepressivo e retardo na recorrência de depressão (Kolouri et al., 2016). Em conjunto o Echium amoenum, M. officinalis (erva-cidreira) e C. sativus (açafrão) demonstraram redução do transtorno depressivo e de complicações significativamente maior que o grupo que utilizou citalopram (Anushiravani et al., 2019).

Outros 3 estudos compararam os efeitos das plantas medicinais ao placebo (Nematolahi et al., 2017; Kyrou et al., 2017; Mazidi et al., 2016;). Nestes, os efeitos das plantas medicinais também foram superiores. Kyrou et al. (2017) ao dispor do Humulus lupulus (lúpulo), concluiu que a utilização do lúpulo sozinho diminuiu de forma significativa os sintomas de depressão, transtorno de humor e ansiedade pela DASS-21, provocando um efeito ansiolítico e possivelmente modulando os níveis dos neurotransmissores gama-aminobutírico (GABA) no Sistema Nervoso Central (SNC). O uso do Rosmarinus officinalis (alecrim) realizado por Nematolahi et al. (2017) teve efeitos na melhora do desempenho da memória, redução da ansiedade e depressão, e melhora na qualidade do sono. O Crocus sativus (açafrão) manifestou efeito significativo na redução da depressão (Mazidi $e t$ al., 2016) (Tabela 2).

Por fim, 3 estudos que utilizaram plantas medicinais adicionadas a medicamentos antidepressivos isolados (Saharaian et al., 2016; Panahi et al., 2015; Talaei et al., 2015). A suplementação de Chlorella vulgaris (CVE) mostrou efeito positivo significativamente superior para os sintomas físicos e cognitivos da depressão (Panahi et al., 2015). Talaei et al. (2015) obteve pontuações significativamente melhores no BDI, BAI e GHQ e a suplementação do C. sativus. (açafrão) apresentou a capacidade de ampliar os efeitos antidepressivos da fluoxetina, citalopram e sertralina. Por outro lado, o estudo de Sahraian et al. (2016) concluiu que o efeito da fluoxetina isolada foi superior ao uso concomitante ao C. sativus (açafrão) (Tabela 2).

\section{Discussão}

Fatores estressores como a duração da quarentena, o medo da infecção, medo da transmissão para familiares, frustração, tédio, informações insuficientes e instabilidade financeira contribuíram para que a depressão tivesse uma grande prevalência durante a pandemia do Covid-19 (Brooks, Webster, Smith, Woodland, Wessely, Greenberg, \& Rubin et al, 2020). Dentre os sintomas mais relatados na população estão: estresse, mau humor, insônia, exaustão e outros (Brooks et al, 2020). Tal contexto motivou o uso de plantas medicinais e/ou fitoterápicos como opções terapêuticas. A grande questão é: quais são os efeitos do uso de plantas medicinais no tratamento de depressão em adultos? Assim, o presente estudo se propôs a identificar e sintetizar as evidências de estudos clínicos controlados sobre os efeitos do uso das plantas medicinais no tratamento de depressão em adultos.

A seleção exclusiva de ensaios clínicos randomizados (ECR) para a revisão deve-se ao fato de que este desenho de estudo é considerado "padrão-ouro" para avaliar a eficácia de intervenções em saúde (Akobeng, 2005). Eles fornecem dados de alta qualidade com menor risco de viés, descrevem a magnitude e precisão do efeito do tratamento, e, a aplicabilidade dos resultados a uma dada população. No entanto, a qualidade metodológica dos ECRs pode ser comprometida pela ausência de alguns critérios, como: alocação dos sujeitos secreta (os pacientes não devem saber se são grupo intervenção ou placebo (Shiwa et al., 2011). Os estudos incluídos nesta revisão possuem notas maiores que 6 pontos na escala de qualidade metodológica e reporte estatístico PEDro. A nota 6 na escala PEDro é considerada a pontuação mínima para a tomada de decisão clínica (Moseley, Elkins, Van der Wees \& Pinheiro, 2020). O critério que mais comprometeu a qualidade foi o da alocação dos sujeitos. Apenas um estudo fez essa alocação secretamente (Nematolahi et al., 2017).

Em relação ao uso das plantas medicinais isoladas ou combinadas com medicamentos antidepressivos, os resultados revelaram efeitos positivos para redução da intensidade dos sintomas de depressão (Araj-Khodaei et al., 2020; Anushiravani $e t$ 
al.,2019; Kolouri et al., 2016), melhora do desempenho da memória e do humor (Kolouri et al., 2016; Panahi et al., 2015), e, melhora na qualidade do sono (Nematolahi et al., 2017). Estes efeitos estiveram relacionados ao uso das plantas por um período que variou entre 4 a 12 semanas.

Os únicos estudos que revelaram efeitos negativos foram os estudos de Sahraian et al. (2016) e de Panahi et al. (2015). Estes estudos utilizaram as plantas medicinais como potencializadores de substâncias já utilizadas para o tratamento da depressão. Sahraian et al. (2016) mediu o efeito do açafrão combinado com a fluoxetina. Panahi et al. (2015) utilizou a suplementação de Chlorella Vulgaris (CVE).

Em síntese, a presente revisão permitiu conhecer os efeitos de plantas medicinais utilizadas no tratamento de sintomas depressivos. Os ECR revelaram a existência de efeitos positivos para uso de plantas medicinais. Apesar dos resultados positivos, alguns efeitos colaterais foram descritos nos estudos. Os mais citados foram: sonolência, náuseas, diarréia, dentre outros. Os efeitos adversos são fatores que devem ser levados em consideração, pois são consequências manifestadas no paciente e que são desconhecidas por quem o está acompanhando no tratamento. Além disso, também é importante considerar algumas limitações metodológicas da revisão. A busca das evidências limitou-se ao uso de bases de dados gratuitas, publicados nos últimos 10 anos, pois essas bases de dados costumam ter trabalhos com uma linguagem mais acessível para a população em geral, sendo de fácil acesso para o leitor desta revisão integrativa.Sugerimos que novos estudos sejam realizados na tentativa de enfrentar as limitações encontradas.

\section{Conclusão}

A presente revisão permitiu conhecer os efeitos de plantas medicinais utilizadas no tratamento de sintomas depressivos. Os resultados revelaram que o uso de plantas medicinais, especialmente da Crocus sativus L. (açafrão), é eficaz para o controle de sintomas depressivos. No entanto, este resultado deve ser interpretado com moderação, em razão do número de estudos e da qualidade metodológica.

\section{Referências}

Akobeng, A. K. (2005). Understanding randomised controlled trials. Archives of disease in childhood, 90(8), $840-844$.

Almeida, C. D. O. (2019). Relatórios de Estágio e Monografia intitulada" Plantas Medicinais no Alívio da Depressão" (Doctoral dissertation, Universidade de Coimbra).

Anushiravani, M., Manteghi, A. A., Taghipur, A., \& Eslami, M. (2019). Comparing effectiveness of a combined herbal drug based on Echium amoenum with Citalopram in the treatment of Major Depressive Disorder. Current drug discovery technologies, 16(2), 232-238.

Araj-Khodaei, (n/a) et al (2020). A double-blind, randomized pilot study for comparison of Melissa officinalis L. and Lavandula angustifolia Mill. with Fluoxetine for the treatment of depression. BMC complementary medicine and therapies, 20(1), 1-9.

Balbino, E. E., \& Dias, M. F. (2010). Farmacovigilância: um passo em direção ao uso racional de plantas medicinais e fitoterápicos. Revista Brasileira de Farmacognosia, 20, 992-1000.

Bueno-Notivol, J. (n/a) et al (2021). Prevalence of depression during the COVID-19 outbreak: A meta-analysis of community-based studies. International journal of clinical and health psychology, 21(1), 100196.

Bruning, M. C. R., Mosegui, G. B. G. \& Vianna, C. M. D. M. (2012). A utilização da fitoterapia e de plantas medicinais em unidades básicas de saúde nos municípios de Cascavel e Foz do Iguaçu-Paraná: a visão dos profissionais de saúde. Ciência \& saúde coletiva, 17, $2675-2685$.

Brooks, S. K., Webster, R. K., Smith, L. E., Woodland, L., Wessely, S., Greenberg, N., \& Rubin, G. J. (2020). The psychological impact of quarantine and how to reduce it: rapid review of the evidence. The lancet, 395(10227), 912-920.

Cleare, A., (n/a) et al (2015). Evidence-based guidelines for treating depressive disorders with antidepressants: a revision of the 2008 British Association for Psychopharmacology guidelines. Journal of Psychopharmacology, 29(5), 459-525.

Fleck, M.P.D.A., Lafer, B., Sougey, E.B., Del Porto, J.A., Brasil, M.A., \& Juruena, M.F. (2003). Diretrizes da Associação Médica Brasileira para o tratamento da depressão (versão integral). Brazilian Journal of Psychiatry, 25, 114-122. 
Galvão, M.C.B. \& Ricarte, I. L. M. (2019). Revisão sistemática da literatura: conceituação, produção e publicação. Logeion: Filosofia da informação, 6(1), 5773 .

Johnson, J., Weissman, M. M., \& Klerman, G. L. (1992). Service utilization and social morbidity associated with depressive symptoms in the community. Jama, 267(11), 1478-1483.

Kessler, R. C., Foster, C. L., Saunders, W. B., \& Stang, P. E. (1995). Social consequences of psychiatric disorders, I: Educational attainment. American journal of psychiatry, 152(7), 1026-1032.

Kolouri, S., Firoozabadi, A., Salehi, A., Zarshenas, M. M., Dastgheib, S. A., Heydari, M., \& Rezaeizadeh, H. (2016). Nepeta menthoides Boiss. \& Buhse freezedried aqueous extract versus sertraline in the treatment of major depression: A double blind randomized controlled trial. Complementary therapies in medicine, 26, 164-170.

Kyrou, I., Christou, A., Panagiotakos, D., Stefanaki, C., Skenderi, K., Katsana, K., \& Tsigos, C. (2017). Effects of a hops (Humulus lupulus L.) dry extract supplement on self-reported depression, anxiety and stress levels in apparently healthy young adults: a randomized, placebo-controlled, double-blind, crossover pilot study. Hormones, 16(2), 171-180.

Luo, Y., (n/a) et al (2018). Evidence synthesis, practice guidelines and real-world prescriptions of new generation antidepressants in the treatment of depression: a protocol for cumulative network meta-analyses and meta-epidemiological study. BMJ open, 8(12), e023222.

Mazidi, M., (n/a) et al (2016). A double-blind, randomized and placebo-controlled trial of Saffron (Crocus sativus L.) in the treatment of anxiety and depression. Journal of Complementary and Integrative Medicine, 13(2), 195-199.

Moseley, A. M., Elkins, M. R., Van der Wees, P. J., \& Pinheiro, M. B. (2020). Using research to guide practice: the physiotherapy evidence database (PEDro). Brazilian journal of physical therapy, 24(5), 384-391.

Nematolahi, P., Mehrabani, M., Karami-Mohajeri, S., \& Dabaghzadeh, F. (2018). Effects of Rosmarinus officinalis L. on memory performance, anxiety, depression, and sleep quality in university students: A randomized clinical trial. Complementary therapies in clinical practice, 30, 24-28.

Panahi, Y., Badeli, R., Karami, G. R., Badeli, Z., \& Sahebkar, A. (2015). A randomized controlled trial of 6-week Chlorella vulgaris supplementation in patients with major depressive disorder. Complementary therapies in medicine, 23(4), 598-602.

Sahraian, A., Jelodar, S., Javid, Z., Mowla, A., \& Ahmadzadeh, L. (2016). Study the effects of saffron on depression and lipid profiles: A double blind comparative study. Asian journal of psychiatry, 22, 174-176.

Shiwa, S. R., Costa, L.O.P., Moser, A.D.D.L., Aguiar, I.D.C., \& Oliveira, L. V. F. D. (2011). PEDro: a base de dados de evidências em fisioterapia. Fisioterapia em Movimento, 24, 523-533.

Talaei, A., Moghadam, M. H., Tabassi, S. A. S., \& Mohajeri, S. A. (2015). Crocin, the main active saffron constituent, as an adjunctive treatment in major depressive disorder: a randomized, double-blind, placebo-controlled, pilot clinical trial. Journal of affective disorders, 174, 51-56.

Whittemore, R., \& Knafl, K. (2005). The integrative review: updated methodology. Journal of advanced nursing, 52(5), 546-553.

World Health Organization. (2013). WHO traditional medicine strategy: 2014-2023. World Health Organization.

Depression, W. H. O. (2017). Other common mental disorders: global health estimates. Geneva: World Health Organization, 1-24.

World Health Organization. (2019). Global status report on alcohol and health 2018. World Health Organization. 